\title{
Differences in Biomechanical Characteristics of Sit-to-Walk Motion between Younger and Elderly Males Dwelling in the Community
}

\author{
MUNETSUGU KOUTA ${ }^{1)}$, KOICHI SHINKODA ${ }^{2)}$ \\ ${ }^{1)}$ Hiroshima City Rehabilitation Center: 1-39-1 Tomominami, Asaminami-Ku, Hiroshima City, \\ 731-3168, Japan. TEL: +81 82-848-8001, FAX: +81 82-848-8003, \\ E-mail: munetsugu@gmail.com \\ ${ }^{2)}$ Hiroshima University Graduate School of Health Sciences
}

\begin{abstract}
Purpose] The purpose of this study was to clarify the difference in sit-to-walk (STW) motion between young adults (YG) and an elderly persons (EG). [Subjects and Methods] Twenty-seven subjects (9 in YG and 18 in EG) were instructed to perform STW and to walk $3 \mathrm{~m}$ at a comfortable speed and at maximal speed. Data were obtained using a 3D motion capture system for kinematic data, and two force plates for kinetic data. [Results] The time required for STW was similar for both groups at both comfortable and maximal speeds, but the forward velocity of the center of gravity of the whole body (COGWB) was higher for YG, and upward COG-WB at the time of the first heel contact was faster for YG. Ground reaction force analyses showed many differences between the groups. For YG, COG-WB moved forward and the first swing side was longer than for EG. [Conclusion] Forward and lateral COG-WB movements before the toe-off generate a progressive force. We suggest that YG subjects may have sufficient muscle power in their lower extremity to bear the progressive force, or that EG subjects may have learned anticipatory postural control which has shorter forward movement due to aging and declining physical function.
\end{abstract}

Key words: Sit-to-Walk, Young and elderly people, Biomechanical analysis

(This article was submitted Mar. 11, 2008, and was accepted May 26, 2008)

\section{INTRODUCTION}

Few researchers have examined the biomechanical characteristics of sit-to-walk motion (STW), although STW is frequently seen in our activities of daily living. In a previous study, Wall et al. ${ }^{1)}$ divided the Timed Up and Go (TUG) test ${ }^{2)}$ into six phases, and analyzed the difference in the duration of each phase between young adults, healthy older adults and older adult fallers. That study pointed out that the sit-to-stand and gait initiation phases were difficult to discern clearly because subjects initiated gait before standing up completely. Kerr et al. ${ }^{3)}$ divided STW into four phases by using ground reaction forces (GRFs) and center of gravity of the whole body (COG-WB). This study defined four phases: flexion momentum, extension, unloading and stance. Magnan et al. ${ }^{4)}$ clarified the difference between STW and sit-tostand (STS) by biomechanically analyzing healthy men. In our previous study of STW ${ }^{5}$, we compared the biomechanics of STW to STS or gait initiation (GI). We found that the maximal horizontal velocity of COG of the trunk (COG-T) before seatoff was significantly faster in STW than in STS. The peak value of backward GRF at the first heel contact was significantly greater in STW than in GI. We suggested that it was important in STW to 
develop forward momentum by bending the trunk quickly, and to brake the forward motion by heel contact.

Generally, elderly people show declines in physical function compared to the young, and elderly fallers show declines in physical function compared to healthy elderly people. The TUG test ${ }^{2}$ ) is useful for evaluating dynamic balance and predicting falls ${ }^{6-8)}$. Although the TUG test includes STW, the biomechanical characteristics of STW in elderly people have not been clarified much. However, there are many comparative studies of gait, STS and GI, which are motions similar to STW $^{9-14)}$. Previous studies have pointed out that there are some differences in STS and GI between the young and elderly, so some differences will also be indicated in STW. If we could clarify the differences between the young and elderly, it would give us a clinical interpretation of the result of the TUG test, and provide a means of clinical intervention for patients who can not achieve STW smoothly.

\section{SUBJECTS AND METHODS}

The subjects were nine healthy young men (young group: YG, mean age $21.8 \pm 2.5$ years) and 18 elderly men (elderly group: EG, mean age $73.9 \pm$ 5.3 years) living at home. Their mean height was $170.3 \pm 4.9 \mathrm{~cm}$ for $Y G$, and $160.0 \pm 4.7 \mathrm{~cm}$ for $E G$; and their mean weight was $65.1 \pm 6.8 \mathrm{~kg}$ for $\mathrm{YG}$, and $56.6 \pm 7.8 \mathrm{~kg}$ for EG. No subject had a disability which restricted his achievement of STW. All elderly subjects were able to do light outdoor work such as cleaning and gardening. Each subject was informed of the purpose of this study, and they all gave their consent to participate in the study. This study was conducted after receiving approval from the ethics committee of Prefectural University of Hiroshima.

The subjects were instructed to perform STW at both comfortable and maximal speeds. STW was initiated from sitting in a chair, and included walking $3 \mathrm{~m}$. A chair without a backrest or armrest was used, and the seat's height was adjusted to match the length of the subject's lower leg. STW was started with the knee flexed at 90 degrees and the feet, shoulder width apart. Subjects started their motion upon hearing a beep emitted by a sound device. The subjects practiced the task until they were able to perform it smoothly and naturally, and
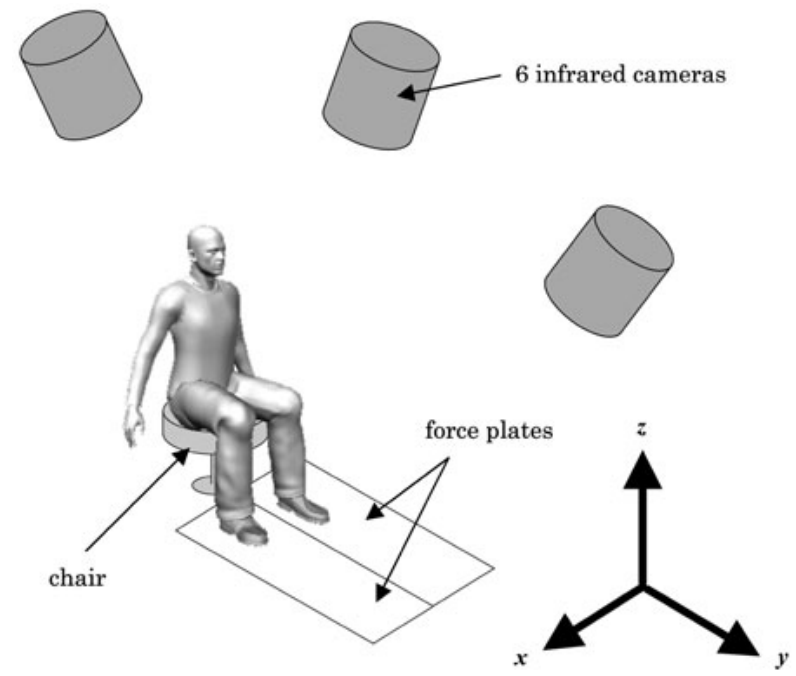

Fig. 1. Measuring system and the three dimensional coordinates.

one trial was adopted for the analysis.

An outline of the experiment is shown in Fig. 1. Kinematic and kinetic data were obtained by a three-dimensional motion capture system (Vicon 512, Oxford Metrix, Ltd.) and two force plates (Kistler force plate 9287BA, $900 \mathrm{~mm} \times 600 \mathrm{~mm}$ ). Kinematic data were obtained by measuring the trajectories of 10 reflective markers (RMs) on landmarks on each subject's body using six infrared cameras. RMs were attached to each landmark on both sides: the acromions, greater trochanters, knee joints, lateral malleoluses, and the metatarsal heads of the fifth toe. Kinetic data were obtained by measuring forces and their coordinates created by both feet on each force plate.

Sampling frequency was $120 \mathrm{~Hz}$ for the infrared cameras and $600 \mathrm{~Hz}$ for the force plates, and force plate data were re-sampled at $120 \mathrm{~Hz}$ by using a Vicon Workstation (ver. 4.6). Kinematic and kinetic data were synchronized after second-order Butterworth low-pass filtering $(6 / 50 \mathrm{~Hz})$. Then, the data were converted to DIFF (Data Interface Format File) using a Vicon Workstation; and DIFF data were converted to Microsoft Excel data (Microsoft Corp.) using "diff gait" and "wave eyes", software produced by The Clinical Gait Analysis Forum of Japan. Coordination of space and GRF defined the $x$ axis as right-left, $y$-axis as forward-backward and $z$-axis as vertical (Fig. 1). Excel data included coordination of RMs and COP, velocity $(V y, V z)$ of COG-WB and COG-T, and GRFs $(F y, F z)$. When 
Table 1. Comparison of the required motion time and the velocity of COG-WB and COG-T

\begin{tabular}{|c|c|c|c|c|c|c|c|}
\hline & & Comforta & speed condit & & Maxim & peed conditic & \\
\hline & & YG & EG & & YG & EG & \\
\hline time required $^{\mathrm{a}}$ & [s] & $1.43 \pm 0.33$ & $1.29 \pm 0.23$ & n.s. & $1.09 \pm 0.13$ & $1.01 \pm 0.10$ & n.s. \\
\hline$V y$ COG-T ${ }^{\mathrm{b}}$ & {$[\mathrm{m} / \mathrm{s}]$} & $0.72 \pm 0.11$ & $0.71 \pm 0.08$ & n.s. & $0.99 \pm 0.20$ & $0.90 \pm 0.16$ & n.s. \\
\hline$V z \mathrm{COG}-\mathrm{WB}^{\mathrm{c}}$ & {$[\mathrm{m} / \mathrm{s}]$} & $0.67 \pm 0.12$ & $0.62 \pm 0.09$ & n.s. & $0.70 \pm 0.15$ & $0.65 \pm 0.12$ & n.s. \\
\hline$V y$ COG-WB ${ }^{\mathrm{d}}$ & {$[\mathrm{m} / \mathrm{s}]$} & $0.37 \pm 0.18$ & $0.36 \pm 0.12$ & n.s. & $0.89 \pm 0.23$ & $0.66 \pm 0.23$ & $*$ \\
\hline
\end{tabular}

$V x, y, z$ : components of velocity; COG-T: center of gravity of the trunk, COG-WB: center of gravity of the whole body. a: the time required from the start of movement of both acromions to the first heel contact; b: maximal $V y$ of COG-T before seat-off; c: maximal $V z$ of COG-WB while standing up; d: $V y$ of COG-WB at the maximal $V z$ of COG-WB while standing up.

n.s.: not significant.

$*$ : $<<0.05$.

Table 2. Upward and anterior velocity of COG-WB while first leg swing

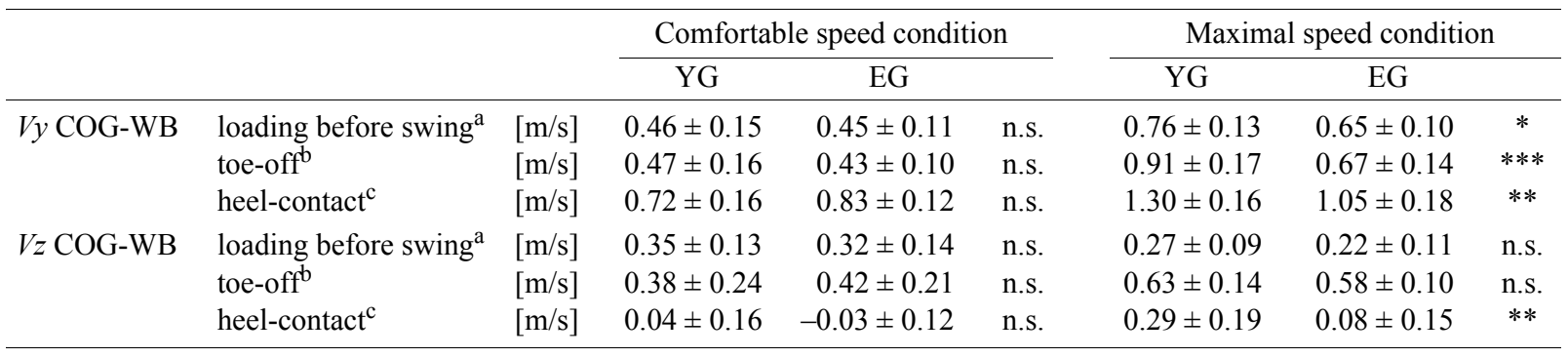

$V x, y, z$ : components of velocity; COG-WB: center of gravity of the whole body.

a: maximal loading on the first swing leg before swing; b: first toe off; c: first heel contact.

n.s.: not significant.

$*: \mathrm{p}<0.05, * *: \mathrm{p}<0.01, * * *: \mathrm{p}<0.001$

forward velocity of both acromions was generated continuously for 0.05 seconds or longer, the start of STW was defined as the time when the motion began.

To compare YG and EG, a non-paired t test was adopted using SPSS ver. 14.0 for Windows (SPSS Japan). The level of statistical significance was set as $\mathrm{p}<0.05$.

\section{RESULTS}

Comparisons between the YG's and EG's required time and velocity of COG are shown in Table 1. The time from the start of STW to the first heel contact was the same for both groups. $V y$ of COG-WB at maximal $V z$ of COG-WB while standing up was significantly faster for the $Y G$ than the EG during the maximal speed $(\mathrm{p}<0.05)$.

To obtain detailed results about the velocity, $V y$ and $V z$ of COG-WB were compared at the time of maximal loading of the swing leg before the first swing, the first toe-off and the first heel-contact
(Table 2). At a comfortable speed there were no significant differences between the two groups. At the maximal speed, $V y$ of COG-WB was higher for YG than for EG at all times, and $V z$ of COG-WB was higher for $Y G$ than for $E G$ at the time of the first heel contact.

GRF analysis showed many significant differences in the groups' maximal GRFs (Table 3 ). At a comfortable speed there were significant differences in all analyzed timings. At the maximal speed, the maximal $F y$ (forward component) was significantly larger for $\mathrm{YG}$ on the first and second stance legs, and the maximal $F z$ was significantly larger for YG at the time of loading the first swing leg before the first swing and the first stance leg.

The distance $(d x, d y, d z)$ between COG-WB and COP at the time of the first toe-off was compared between the groups (Table 4). $d x$ was indicated as the first swing side to COP. The results showed no significant differences at comfortable speeds. At the maximal speed, $d x$ and $d y$ were significantly longer for $\mathrm{YG}$ than $\mathrm{EG}$, and $d z$ was significantly 
Table 3. Comparison of GRFs between YG and EG

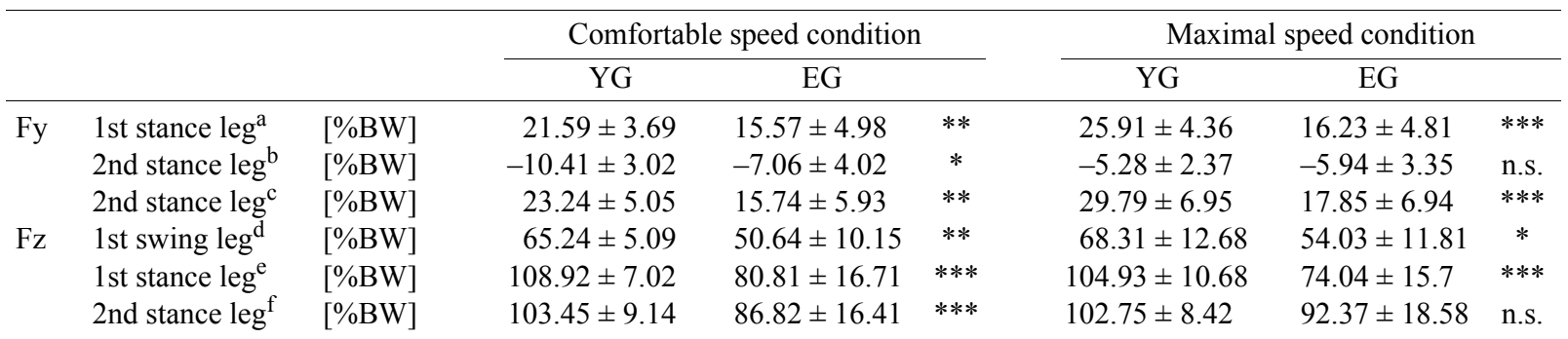

a: maximal forward GRF on the first stance leg; b: maximal backward GRF on the second stance leg; c: maximal forward GRF on the second stance leg; d: maximal upward GRF on the first swing leg before swing; e: maximal upward GRF on the first stance leg; f: maximal upward GRF on the second stance leg. n.s.: not significant.

$*: \mathrm{p}<0.05, * *: \mathrm{p}<0.01, * * *: \mathrm{p}<0.001$
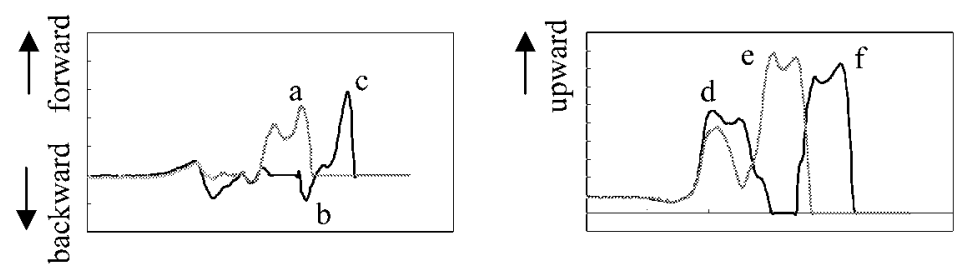

Table 4. Distance between COG-WB and COP

\begin{tabular}{|c|c|c|c|c|c|c|}
\hline & \multicolumn{3}{|c|}{ Comfortable speed condition } & \multicolumn{3}{|c|}{ Maximal speed condition } \\
\hline & YG & EG & & $\mathrm{YG}$ & EG & \\
\hline$[\% \mathrm{BH}]$ & $5.07 \pm 0.56$ & $4.30 \pm 1.10$ & n.s. & $5.48 \pm 1.03$ & $4.56 \pm 1.09$ & $*$ \\
\hline dy $[\% \mathrm{BH}]$ & $6.68 \pm 2.06$ & $5.50 \pm 1.73$ & n.s. & $9.71 \pm 9.59$ & $7.50 \pm 2.15$ & $*$ \\
\hline $\mathrm{dz} \quad[\% \mathrm{BH}]$ & $53.9 \pm 3.24$ & $52.8 \pm 3.30$ & n.s. & $47.2 \pm 3.77$ & $49.9 \pm 2.76$ & $*$ \\
\hline
\end{tabular}

$\mathrm{dx}, \mathrm{y}, \mathrm{z}$ : components of distance between COG-WB and COP.

n.s: not significant, $*$ : $\mathrm{p}<0.05$.

longer for EG than YG.

\section{DISCUSSION}

This study analyzed biomechanical STW characteristics between YG and EG. The time required did not differ significantly between the groups at comfortable and maximal speeds (Table 1). Maximal $V y$ of COG-T before the seat-off, and maximal $V z$ of COG-WB while standing, did not differ significantly between the groups. Mourey et al. ${ }^{12)}$ reported, in their STS study, that maximal forward velocity and maximal composite velocity of COG-WB during the seat-off were larger in YG than EG at both comfortable and maximal speeds. This study found no difference in the velocity of COG before the seat-off and while standing up between the groups because this study included subjects in EG who had better physical functions.

At the maximal speed condition, $V y$ of COG-WB at all analyzed times was faster for YG than EG (Table 2), although the required time from the starting movement to the first heel contact didn't differ significantly between the groups. It therefore seems that the difference in the required time might become larger after the first heel contact. $V z$ of COG-WB at the first heel contact was significantly faster for $Y G$ than EG at the maximal speed. At this time YG continued standing up, while EG had already achieved an upright standing position. It seems that initiating gait before achieving an upright standing position was difficult for EG.

This study found many significant differences in GRFs between YG and EG. All components of GRFs which differed significantly between the groups showed a higher value for YG (Table 3). At a comfortable speed, although no significant differences were shown in the vertical and forward velocities of COG-WB and COG-T between the two groups (Table 2), the results do show significant differences in all vertical and forwardbackward GRFs between them (Table 3). This inconsistency may have occurred because of quick and effective force generation of the leg, followed 
by smooth force transmission to the COG. In previous studies, patients with Parkinson's disease stood up with GRF values lower than those of agematched healthy adults, although the required time did not differ between the two groups ${ }^{15)}$. Tokumo et al. ${ }^{16)}$ reported that people who had had a stroke initiated their gait with forward-backward GRF value lower than healthy older adults, although their motion was adjusted and performed at the same speed. In this study, it seems that YG could perform the STW motion by extending their legs quickly.

In the relationship between COG-WB and COP at the first toe-off, COG-WB moved farther forward and to the first swing side in YG. In contrast, COGWB moved farther upward in EG (Table 4). On gait initiation, COG-WB movement before the first toeoff was important for anticipatory postural control $^{17)}$. If the speed of initiating gait is changed, the movement of COG-WB as a predictor of postural control is changed ${ }^{18)}$. So it is thought that EG can /do not have to move COG-WB forward, and their first swing side is farther. Forward and lateral COG-WB movement before toe-off makes the movement a progressive force. It can be said that YG have sufficient muscle power in their lower extremities to bear the progressive force, or that EG subjects may have learned an appropriate anticipatory postural control in which they move forward a shorter distance due to aging and declining physical function.

\section{REFERENCES}

1) Wall JC, Bell C, Campbell S, et al.: The timed get-upand-go test revisited: measurement of the component tasks. J Rehabil Res Dev, 2000, 37: 109-113.

2) Podsiadlo D, Richardson S: The timed "up \& go": A test of basic functional mobility for frail elderly persons. J Am Geriatr Soc, 1991, 39: 142-148.

3) Kerr A, Durward B, Kerr KM: Defining phases for the sit-to-walk movement. Clin Biomech, 2004, 19: 385390 .

4) Magnan A, McFadyen BJ, St-Vincent G: Modification of the sit-to-stand task with the addition of gait initiation. Gait Posture, 1996, 4: 232-241.

5) Kouta M, Shinkoda K, Kanemura N: Sit-to-walk versus sit-to-stand or gait initiation: Biomechanical analysis of young men. J Phys Ther Sci, 2006, 18: 201-206.

6) Newton RA: Balance screening of an inner city older adult population. Arch Phys Med Rehabil, 1997, 78: 587-591.

7) Chiu AY, Au-Yeung SS, Lo SK: A comparison of four functional tests in discriminating fallers from nonfallers in older people. Disabil Rehabil, 2003, 25: 4550.

8) Hansen K, Mahoney J, Palta M: Risk factors for lack of recovery of $\mathrm{ADL}$ independence after hospital discharge. J Am Geriatr Soc, 1999, 47: 360-365.

9) Henriksson M, Hirschfeld H: Physically active older adults display alterations in gait initiation. Gait Posture, 2005, 21: 289-296.

10) Mickelborough J, van der Linden ML, Tallis RC, et al.: Muscle activity during gait initiation in normal elderly people. Gait Posture, 2004, 19: 50-57.

11) Martin M, Shinberg M, Kuchibhatla M, et al.: Gait initiation in community-dwelling adults with Parkinson disease: comparison with older and younger adults without the disease. Phys Ther, 2002, 82: 566577.

12) Mourey F, Grishin A, d'Athis P, et al.: Standing up from a chair as a dynamic equilibrium task: a comparison between young and elderly subjects. J Gerontol A Biol Sci Med Sci, 2000, 55: 425-431.

13) Papa E, Cappozzo A: Sit-to-stand motor strategies investigated in able-bodied young and elderly subjects. J Biomech, 2000, 33: 1113-1122.

14) Lord SR, Murray SM, Chapman K, et al: Sit-to-stand performance depends on sensation, speed, balance, and psychological status in addition to strength in older people. J Gerontol A Biol Sci Med Sci, 2002, 57: 539543.

15) Ramsey VK, Miszko TA, Horvat M: Muscle activation and force production in Parkinson's patients during sit to stand transfers. Clin Biomech, 2004, 19: 377-384.

16) Tokuno CD, Eng JJ: Gait initiation is dependent on the function of the paretic trailing limb in individuals with stroke. Gait Posture, 2006, 24: 424-428.

17) Ito T, Azuma T, Yamashita N: Anticipatory control in the initiation of a single step under biomechanical constraints in humans. Neurosci Lett, 2003, 352: $207-$ 210.

18) Gelat $T$, Breniere $Y$ : Adaptation of the gait initiation process for stepping on to a new level using a single step. Exp Brain Res, 2000, 133: 538-546. 